\title{
A central-peripheral asymmetry in masked priming
}

\author{
FRIEDERIKE SCHLAGHECKEN and MARTIN EIMER \\ Birkbeck College, London, England
}

\begin{abstract}
Masked primes presented prior to a target result in behavioral benefits on incompatible trials (in which the prime and the target are mapped onto opposite responses) when they appear at fixation, but in behavioral benefits on compatible trials (in which the prime and the target are mapped onto the same response) when appearing peripherally. In Experiment 1, the time course of this centralperipheral asymmetry (CPA) was investigated. For central primes, compatible-trial benefits at short stimulus onset asynchronies (SOAs) turned into incompatible-trial benefits at longer SOAs. For peripheral primes, compatible-trial benefits at short SOAs increased in size with longer SOAs. Experiment 2 showed that these effects also occur when primes and targets are physically dissimilar, ruling out an interpretation in terms of the perceptual properties of the stimulus material. In Experiments 3 and 4 , the question was investigated as to whether the CPA is related to visual-spatial attention and/or retinal eccentricity per se. The results indicate that the CPA is independent of attentional factors but strongly related to the physiological inhomogeneity of the retina. It is argued that central and peripheral primes trigger an initial motor activation, which is inhibited only if primes are presented at retinal locations of sufficiently high perceptual sensitivity. The results are discussed in terms of an activation threshold model.
\end{abstract}

In the last decade, the role of inhibition in cognitive processes has received considerable attention. On the basis of evidence from different experimental paradigms, the concept of inhibitory control of information processing is gaining increasing support. Possible examples of inhibitory control currently under discussion include inhibitory processing in the perception and/or encoding of repeated items (repetition blindness; Kanwisher, 1987); an inhibitory bias in spatial attention (inhibition of return; Maylor, 1985), the inhibition of visual processing as a result of target identification (attentional blink, Raymond, Shapiro, \& Arnell, 1992), the inhibition of irrelevant objects during target selection (negative priming; Tipper, 1985; see Fox, 1995, and May, Kane, \& Hasher, 1995, for reviews), and the selective inhibition of ongoing motor activity (inhibitory motor control; De Jong, Coles, \& Logan, 1995; Kopp, Rist, \& Mattler, 1996). Although the specific mechanisms underlying these phenomena may differ, they are likely to reflect the operation of adaptively significant inhibitory control processes, rather than just basic capacity limitations of the cognitive system (see Arbuthnott, 1995, and Houghton \& Tipper, 1996, for further discussions).

An area in which inhibitory effects generally have not been found is the field of perception without subjective awareness, or near-threshold perception (for a notable ex-

The present research was supported by grants from the Biotechnology and Biological Sciences Research Council and from the Deutsche Forschungsgemeinschaft. The authors thank Arno von Buxhoeveden and Jutta Braune for their help in running the experiments. Correspondence concerning this article should be addressed to F. Schlaghecken, Department of Experimental Psychology, Birkbeck College, Malet Street, London WC1E, 7HX, England (e-mail: f.schlaghecken@bbk. ac.uk). ception, see Dagenbach, Carr, \& Wilhelmsen, 1989). For example, inhibiting the attentional shift toward the location of a highly invalid peripheral cue is possible with suprathreshold cues, but impossible if cues are presented near detection threshold (McCormick, 1997). Similarly, inhibiting one of two meanings of a polysemous word is possible for suprathreshold words, but not for nearthreshold words (Marcel, 1980). Finally, negative priming effects have been found to turn into positive priming effects when probe-trial distractors were successfully masked (Allport, Tipper, \& Chmiel, 1985; see also Neill, Valdes, \& Terry, 1995).

Recently, however, evidence has been reported that even under conditions of near-threshold stimulus presentation, inhibitory processes can be observed (Eimer, 1999; Eimer \& Schlaghecken, 1998; Schlaghecken \& Eimer, 1997). In the masked prime paradigm, participants had to perform a choice reaction time (RT) task in response to simple visual stimuli, such as arrows or geometric figures, that were preceded by masked primes. ${ }^{1}$ In compatible trials, the prime and the target were identical; in incompatible trials, they were mapped to different responses; in neutral trials, the prime was task irrelevant. Surprisingly, performance benefits (fast responses, low error rates) were obtained in incompatible trials, whereas performance costs were present in compatible trials.

First insights into the basis of this unexpected negative compatibility effect were obtained by inspecting the lateralized readiness potential (LRP), an electrophysiological measure of unimanual response activation (Eimer, 1999; Eimer \& Schlaghecken, 1998): Around $200 \mathrm{msec}$ after prime onset, the LRP showed a partial activation of the response assigned to the prime stimulus. However, about $100 \mathrm{msec}$ later, in the time range in which responses 
to the target stimulus were selected and activated, this initial response activation was replaced by an activation of the opposite response. It was argued that the early partial activation of the response assigned to the prime is a consequence of direct perceptuomotor links, whereas the subsequent reversal phase reflects an active inhibition of this initial response tendency. The negative compatibility effect on behavioral performance would thus reflect an inhibition of a response tendency triggered by the masked primes. This activation-followed-by-inhibition hypothesis can also account for the fact that performance benefits for compatible trials (positive compatibility effects) were observed in experiments in which the masking stimulus itself served as the target (Klotz \& Wolff, 1995; Neumann \& Klotz, 1994): When the target immediately follows the prime instead of being separated from the prime by a masking stimulus, prime-target stimulus onset asynchronies (SOAs) are shorter, so that the activation of the response to the target is more likely to overlap with the initial, prime-related partial response activation. This would result in performance benefits in compatible trials, owing to the partial activation of the correct response, and in costs in incompatible trials, owing to the partial activation of the incorrect response.

In accordance with the activation-followed-by-inhibition account, experiments that varied the SOA between mask and target (Eimer, 1999, Experiment 3; Schlaghecken \& Eimer, 1997) found positive compatibility effects for a mask-target SOA of $0 \mathrm{msec}$, but these effects decreased with longer SOAs and eventually turned into negative compatibility effects. Moreover, negative compatibility effects were also obtained when responses with two fingers of the same hand were required and even when only a single overt response was to be executed in a go/no-go experiment (Eimer \& Schlaghecken, 1998, Experiment 3): When go stimuli were delivered as primes, responses to go targets were delayed and false alarms to no-go stimuli were less frequent, relative to trials in which a no-go stimulus served as the prime, suggesting that the response assigned to the go prime was actively inhibited.

In the studies described above, behavioral and electrophysiological evidence for activation-followed-byinhibition was found when masked primes were presented at fixation. However, with masked primes presented in the periphery, a very different pattern of results emerged. Schlaghecken and Eimer (1997) compared the behavioral effects of foveally and peripherally presented masked primes and found that with a 96-msec mask-target SOA, central primes elicited negative compatibility effects, whereas peripheral primes elicited positive compatibility effects (i.e., performance benefits for compatible trials).

How does this central-peripheral asymmetry (CPA) fit into the activation-followed-by-inhibition framework? A comparable situation, in which the influence of (unmasked) distractor stimuli on responses to targets varies with the spatial separation of target and distractors, can be found in the flanker compatibility paradigm (e.g., B. A. Eriksen \& C. W. Eriksen, 1974): Similar to the masked prime paradigm, psychophysiological evidence indicates that this interference is due to the fact that distractors that are potential target stimuli are likely to trigger a partial activation of their corresponding motor response (Gratton, Coles, Sirevaag, Eriksen, \& Donchin, 1988; Smid, Mulder, \& Mulder, 1990). In addition, similar to the masked prime paradigm, the interference effect changes with changing retinal eccentricity of the distractors (e.g., C. W. Eriksen \& Hoffmann, 1973; C. W. Eriksen, Pan, \& Botella, 1993; Goolkasian, 1997; Miller, 1991). However, in the masked prime paradigm, increased retinal eccentricity of the distractors resulted in a reversal of the negative compatibility effect, whereas in the flanker compatibility paradigm, only a gradual decrease in interference effects has been observed, without evidence for "a different kind of processing" (Goolkasian, 1997) of central and peripheral information.

The present experiments were conducted to investigate whether the CPA in prime-target compatibility effects reflects qualitatively different types of processing for central and peripheral stimuli. If it could be established that there are systematic differences in the way that foveal information and peripheral information affect motor processes, this may have implications for our understanding of the mechanisms mediating between perception and action and of the role of inhibitory processes in perceptuomotor links. Experiment 1 was conducted to confirm that the CPA is a reliable effect in masked priming situations and to investigate the time course of the effects of central and peripheral primes on behavioral performance. Experiment 2 was conducted to rule out the idea that the CPA is primarily based on perceptual properties of the stimuli employed previously, rather than reflecting different patterns of response activation and inhibition. Experiments 3 and 4 studied the relation between spatial attention and the CPA, as well as the role of physiological properties of the visual system correlated with retinal eccentricity.

\section{EXPERIMENT 1}

Experiment 1 was conducted to test whether primetarget compatibility effects elicited by central and peripheral masked primes reflect the same underlying processes, which vary only in their latencies, or whether they reflect qualitatively different processes. The onset of the partial response activation triggered by the prime may simply be delayed for peripheral relative to foveally presented primes - for example, because perceptual analysis takes longer for peripherally presented information than for centrally presented information (see Coyle, 1994). If this were the case, then with peripheral primes, the activation of the response required by the target would be more likely to overlap with the initial, prime-related activation, instead of occurring during the subsequent response inhibition phase. In Experiment 1A, prime-target compatibility effects for central and peripheral primes were investigated with short to intermediate mask-target 
SOAs (0-96 msec). If the CPA is due to a delayed response activation triggered by peripheral primes, then with a 0 -msec SOA, no compatibility effects should be elicited by peripheral primes while central primes should give rise to a positive compatibility effect. Increasing the SOA should result in gradually emerging positive compatibility effects for peripheral primes and in a reversal from positive to negative compatibility effects with central primes. ${ }^{2}$

Alternatively, the onset of the inhibition phase may be delayed with peripheral primes, causing prime-target compatibility effects to reverse polarity later for peripherally than for centrally presented primes. In Experiment $1 \mathrm{~B}$, prime-target compatibility effects were investigated with intermediate to long SOAs (96-192 msec) in order to test whether peripherally presented primes would give rise to negative compatibility effects at longer SOAs. Moreover, the possibility that the negative compatibility effect elicited by central primes would remain stable with longer mask-target SOAs was tested. If, however, positive compatibility effects were observed for peripheral primes at all SOAs, this would indicate that inhibitory processes may be entirely restricted to response tendencies elicited by foveally presented information, whereas response activations triggered by peripheral information may not be actively inhibited at all.

\section{Method}

Participants. Twelve paid volunteers ( 5 males), $21-37$ years of age (mean age, 29.1 years), participated in Experiment $1 \mathrm{~A}$, and 12 paid volunteers (6 males), 18-38 years of age (mean age, 28.7 years), participated in Experiment 1B. All the participants were right-handed and had normal or corrected-to-normal vision.

Stimuli and Apparatus. Left- and right-pointing double arrows $(<<$ and $>>)$ served as prime stimuli. The mask was constructed by superimposing these stimuli upon one another. The target consisted of two left- or right-pointing double arrows $(<<<<$ and $>>>>)$, spaced appropriately to allow the prime and the mask to fit exactly into the central gap. All the stimuli were presented in black on a white background on a 17-in. computer screen. Prime and mask stimuli subtended a visual angle of approximately $1.15^{\circ} \times 0.4^{\circ}$; target stimuli subtended a visual angle of approximately $3.45^{\circ} \times 0.4^{\circ}$.

Procedure. The participants were seated in a dimly lit, soundattenuated chamber, with response buttons under their left and right index fingers. A computer screen was placed $100 \mathrm{~cm}$ in front of the participants' eyes so that the screen center was in the center of the participants' horizontal straight-ahead line of sight. The participants were instructed to maintain central eye fixation and to respond as fast and accurately as possible with a left buttonpress to a left-pointing target arrow and with a right buttonpress to a right-pointing target arrow.

Experimental blocks consisted of 80 trials, each starting with the presentation of a prime stimulus (16-msec duration), followed by a mask (100-msec duration) and a target stimulus (100-msec duration). In the central condition, masked primes appeared at fixation. In the peripheral condition, masked primes appeared randomly and with equal probability either $2.8^{\circ}$ above or $2.8^{\circ}$ below fixation. Target stimuli appeared always to the left and right of fixation (see above). Trials were termed compatible when prime and target arrows were pointing in the same direction, and incompatible otherwise. Both conditions were equiprobable and randomized within a given block.
The mask always followed the prime immediately, whereas masktarget SOA was blocked and was 0, 32, 64, or $96 \mathrm{msec}$ (Experiment $1 \mathrm{~A}$ ) or $96,128,160$, or $192 \mathrm{msec}$ (Experiment 1B). The intertrial interval was $1 \mathrm{sec}$. Different prime locations (central and peripheral) were also blocked. Since each of the four SOAs was combined with each of the two prime locations, there was a total of eight different location/SOA combinations in each experiment. Each location/SOA combination was delivered in four blocks, resulting in a total of 32 experimental blocks per experiment. The experiments were divided into four parts, each containing one block of each location/SOA combination. Within each part, the order in which these blocks were delivered was randomized. ${ }^{3}$

Data analysis. Repeated measures analyses of variance (ANOVAs) were performed on RTs and error rates in the experimental blocks for the factors of prime position (central or peripheral), $\operatorname{SOA}(0,32$, 64 , or $96 \mathrm{msec}$ in Experiment $1 \mathrm{~A}$ and $96,128,160$, or $192 \mathrm{msec}$ in Experiment 1B), compatibility (compatible or incompatible), and response side (left or right). Where appropriate, Greenhouse-Geisser adjustments to the degrees of freedom were performed (indicated in the Results section by $\varepsilon$ ).

\section{Results}

In both experiments, main effects of prime position were obtained for RTs and error rates [all $F_{\mathrm{s}}(1,11)>8.7$, all $p$ s $<.013$ ], since RTs were shorter and fewer errors were made in the peripheral prime condition $(372 \mathrm{msec}$, $1.9 \%$ errors in Experiment $1 \mathrm{~A}$, and $358 \mathrm{msec}, 1.9 \%$ in Experiment 1B) than in the central prime condition $(396 \mathrm{msec}$, $2.7 \%$, and $377 \mathrm{msec}, 2.9 \%$, respectively).

Experiment 1A. Overall, RTs were shorter and fewer errors were made in compatible trials than in incompatible trials $[377 \mathrm{msec}, 1.8 \%$ vs. $391 \mathrm{msec}, 2.9 \% ; F(1,11)=$ 63.04 and 31.59 , both $p \mathrm{~s}<.001$, for RT and error rates, respectively]. For RT, this effect was larger for peripheral primes than for central primes $[F(1,11)=11.98, p<$ $.005]$, and for both RTs and error rates positive compatibility effects were more pronounced with short SOAs than with long SOAs [RT: $F(3,33)=19.20, p<.001, \varepsilon=$ .694 ; error rates: $F(3,33)=11.27, p<.001, \varepsilon=.827]$. As can be seem from Figure 1, the positive compatibility effect not only became smaller with increasing SOA for central primes, but turned into a negative compatibility effect with the 96-msec SOA. In contrast, positive compatibility effects became larger with increasing SOAs for peripheral primes. This pattern was reflected in prime position $\times$ SOA $\times$ compatibility interactions for RTs and error rates $[F(3,33)=35.07$ and 18.5 , both $p s<$ $.001, \varepsilon=.590$ and .669 , respectively].

Pairwise comparisons of RTs for compatible and incompatible trials, conducted with two-tailed $t$ tests for individual SOAs and prime positions, confirmed that for peripheral primes, RT for compatible trials was significantly shorter than RT for incompatible trials in all SOA conditions [all $t \mathrm{~s}(11)>5.9$, all $p \mathrm{~s}<.001$ ]. For central primes, RT for compatible trials was significantly shorter than RT for incompatible trials in the 0 -msec SOA condition and in the 32-msec SOA condition [both $t \mathrm{~s}(11)>6.4$, both $p s<.001$ ], whereas it was significantly longer in the $96-\mathrm{msec}$ SOA condition $[t(11)=4.06, p<.002]$. No 


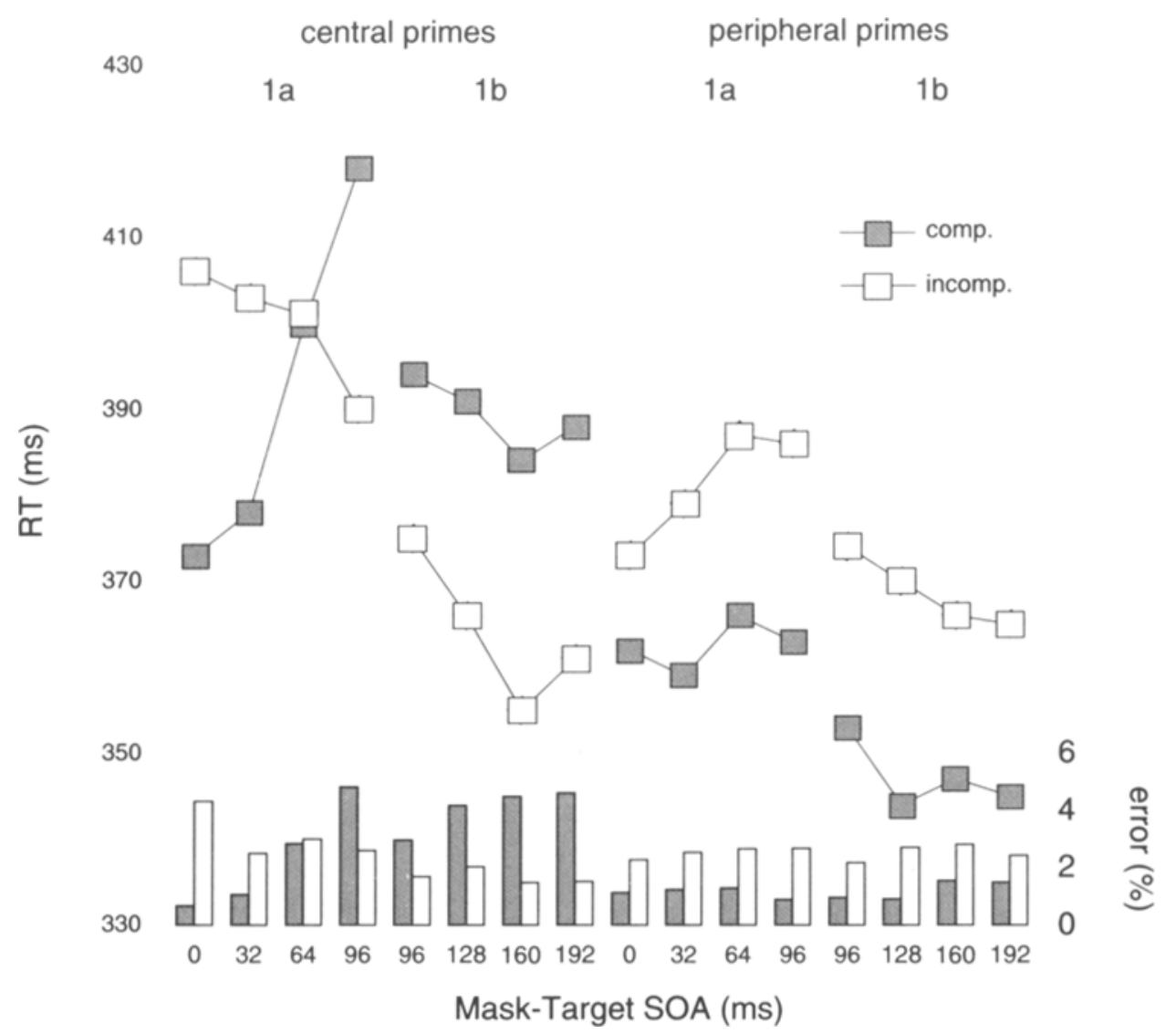

Figure 1. Experiment 1: Reaction times (RTs; line graphs) and error rates (bar graphs) in compatible trials (gray) and incompatible trials (white) for the four stimulus onset asynchrony (SOA) conditions in Experiment $1 \mathrm{~A}(0,32,64$, and $96 \mathrm{msec})$ and Experiment $1 \mathrm{~B}(96,128,160$, and $192 \mathrm{msec})$, displayed separately for central primes (left) and peripheral primes (right).

significant compatibility effect was present for central primes in the 64-msec SOA condition.

Experiment 1B. No main effect of compatibility was present, but highly significant compatibility $\times$ prime position interactions for RTs and error rates $[F(1,11)=$ 31.82 and 19.66 , respectively; both $p s<.001$ ] indicated the presence of prime-target compatibility effects of opposite directions for the two prime positions (see Figure 1). With peripheral primes, a stable positive compatibility effect was found, whereas with central primes, a stable negative compatibility effect was observed. Additional ANOVAs, conducted separately for central trials and peripheral trials, confirmed that these effects were significant for peripheral primes [RT: $F(1,11)=73.20$, $p<.001$; error rate: $F(1,11)=15.10, p<.003]$, as well as for central primes [RT: $F(1,11)=14.13, p<.003$; error rate: $F(1,11)=8.42, p<.014]$. Importantly, there was no compatibility $\times$ SOA interaction on either RT or error rates for either central or peripheral primes, indicating that the respective priming effects were of equivalent size across all SOAs.

\section{Discussion of Experiment 1}

The effect of masked prime stimuli on performance to subsequently presented targets depends crucially on the location of these primes. With mask-target SOAs of $96 \mathrm{msec}$, negative compatibility effects were observed with centrally presented primes, but positive compatibility effects were present for peripheral primes (Schlaghecken \& Eimer, 1997). Experiment 1 was conducted to test whether this CPA was due to latency differences in the response activation and/or inhibition processes triggered by central and peripheral primes. To this purpose, the time course of priming effects was investigated for central and peripheral masked primes by varying masktarget SOA between 0 and $192 \mathrm{msec}$.

The results confirmed that the CPA is a reliable phenomenon that can be obtained under a number of different SOA conditions. None of these conditions, however, revealed any evidence that the CPA is due to latency differences: In Experiment 1A, peripheral primes produced a substantial positive compatibility effect in the $0-\mathrm{msec}$ SOA condition, indicating that the onset of response ten- 
dencies triggered by peripheral primes was not (or not substantially) delayed, as compared with response tendencies triggered by central primes. In Experiment 1B, both positive compatibility effects obtained with peripheral primes and negative compatibility effects obtained with central primes remained constant across the whole range of SOAs employed in this study. This suggests that response inhibition elicited in the central prime condition is not a transient phenomenon that is subject to rapid decay, whereas response inhibition is entirely absent in the peripheral prime condition. Although response inhibition with peripheral primes may have been obtained at even longer SOAs, the fact that the positive compatibility effects showed no sign of decrease with increasing SOA makes this assumption unlikely.

However, it has to be noted that the positive compatibility effect obtained for the 0 -msec SOA was significantly smaller for peripheral than for central primes [12 vs. $33 \mathrm{msec} ; t(11)=4.47, p<.001$ ]. This difference may indicate that during the response activation for a 0 -msec SOA target, the response tendency triggered by central primes was already fully activated, whereas it was only just beginning to build up for peripheral primes. If this were the case, positive compatibility effects in response to peripheral primes in the 0 -msec SOA condition should be restricted to relatively slow responses, because faster responses would be selected and activated prior to the onset of the prime-related response activation. This was tested by comparing RT effects for peripheral primes in the 0 -msec SOA condition for the fastest $10 \%$ of all responses (mean RT of about $300 \mathrm{msec}$ ) with the effects obtained for the slowest $10 \%$ of all responses (mean RT of about $415 \mathrm{msec}$ ), obtained on the basis of individual RT distributions. Positive compatibility effects were obtained for slow responses $[t(11)=2.97, p<.013]$, as well as for fast responses $[t(11)=4.62, p<.001]$, and these effects did not differ in size $[t(11)<1.3$, n.s.]. Assuming that the selection and activation of the response to the target precedes execution by at least $100 \mathrm{msec}$, the fact that positive compatibility effects were present for the fastest responses in the 0 -msec SOA condition indicates that peripheral primes affected motor activation around $200 \mathrm{msec}$ after their onset. This estimate corresponds well with LRP evidence obtained with central primes (Eimer \& Schlaghecken, 1998), demonstrating that the partial activation of the primed response starts about $200 \mathrm{msec}$ after prime onset, and suggests that the onset of the primed response activation is not systematically delayed for peripheral relative to central primes.

Taken together, the results of Experiment 1 support the idea that there are qualitative differences in response activation processes triggered by central and peripheral primes: Whereas central primes give rise to an activationfollowed-by-inhibition process, peripheral primes seem to elicit a process of activation-only. Since this conclusion may have important implications for our understanding of how visual information affects performance, alternative interpretations and possible confounding factors have to be carefully considered. In Experiment 2, the question of whether the CPA is based on perceptual differences that are due to stimulus presentation was investigated: Perhaps the rapid succession of central primes and targets has resulted in specific emergent features that did not occur with peripheral primes. Experiments 3 and 4 addressed the question of attentional differences: Because all the targets were presented at fixation, central primes were processed within the focus of attention, whereas peripheral primes, presented at unattended locations, were not.

\section{EXPERIMENT 2}

In Experiment 1, prime and target arrows were identical in compatible trials, while pointing in opposite directions in incompatible trials. The successive presentation of primes and targets at adjacent locations in the central presentation condition may have resulted in qualitatively different emergent visual features in compatible and incompatible trials, resulting in an advantage for incompatible trials in the central presentation condition. Owing to the spatial separation between primes and targets, no such emergent features would be elicited in the peripheral presentation condition. In addition, the presentation of two identical stimuli in rapid succession and close spatial proximity may impair the detection of the second stimulus, relative to a situation in which these stimuli are not identical or appear at different locations (perceptual repetition blindness; see Hochhaus \& Johnston, 1996). If this phenomenon was responsible for the negative compatibility effects obtained with central primes and longer SOAs in Experiment 1, this may fully explain the presence of a CPA. To investigate these issues, physically similar, as well as physically dissimilar, prime and target stimuli were employed in Experiment 2. If the CPA was based on perceptual properties of the stimuli employed in Experiment 1 , it should be obtained in the similar condition but should disappear in the dissimilar condition.

\section{Method}

Participants. Eight paid volunteers ( 7 female), 19-39 years of age (mean age, 26.5 years), participated in the experiment. All the participants were right-handed and had normal or corrected-tonormal vision.

Stimuli, Apparatus, and Procedure. The stimuli, apparatus, and procedure were identical to the 0 - and $128-\mathrm{msec}$ SOA conditions of Experiments $1 \mathrm{~A}$ and $1 \mathrm{~B}$, respectively, except that different target stimuli were employed. In $50 \%$ of all the trials, central arrow targets were presented, whereas in the other half, lateral target stimuli were delivered. Central targets consisted of two left-pointing or rightpointing double arrows, appearing bilaterally $1.5^{\circ}$ above and below fixation $^{4}$ (measured from center to center) and spaced appropriately as to to allow central primes and masks to fit exactly into the central gap. Lateral targets were single plus signs $(+)$, subtending a visual angle of approximately $0.3^{\circ} \times 0.3^{\circ}$ that appeared unilaterally at a horizontal distance of $2.4^{\circ}$ to the left or right of fixation. Central and lateral targets were presented in randomized order. ${ }^{5}$

The participants were instructed to respond with a left keypress to left-pointing central target arrows and to lateral targets appearing at the left side and with a right keypress to right-pointing central target arrows and to lateral targets appearing at the right side. Trials were termed compatible when prime and target arrows were pointing in the same direction or when a plus-target appeared at the 
side indicated by the prime and incompatible otherwise. Twenty experimental blocks consisting of 80 trials each were run. Prime location (central vs. peripheral) and mask-target SOA (0 vs. $128 \mathrm{msec})$ were blocked, with five successive blocks for each of the four combinations of these conditions. At the beginning of each of these four experimental segments, a brief practice block was delivered.

Data analysis. Repeated measures ANOVAs were performed on mean RTs and error rates, separately for central and lateral targets, for the factors of prime position (central or peripheral), SOA (0 or $128 \mathrm{msec}$ ), compatibility (compatible or incompatible), and response side (left or right). Follow-up analyses were included to investigate interactions between these factors.

\section{Results}

Figure 2 shows the mean RTs and error rates obtained in compatible and incompatible trials in the 0 - and 128msec mask-target SOA conditions with central and peripheral primes and central and lateral targets, respectively. Overall, responses were faster to lateral targets than to central targets and, similar to Experiment 1, faster on peripheral prime trials than on central prime trials.

For both target types, responses were generally faster and fewer errors were made on compatible trials than on incompatible trials [RT: $F \mathrm{~s}(1,7)=10.09$ and $8.29, p \mathrm{~s}<$
.016 and .024 , for central and lateral targets, respectively; error rates: $F_{\mathrm{s}}(1,7)=16.45$ and $19.44, p \mathrm{~s}<.006$ and $.003]$. However, these effects were accompanied by prime position $\times$ compatibility interactions $\left[\mathrm{RT}: F_{\mathrm{S}}(1,7)=39.4\right.$ and $13.52, p \mathrm{~s}<.001$ and .008 ; error rates, central targets: $F(1,7)=26.27, p<.001$; lateral targets: $F(1,7)=2.33$, n.s.], since positive compatibility effects were much larger on peripheral prime trials than on central prime trials. Most important, they were accompanied by threeway prime position $\times$ SOA $\times$ compatibility interactions [RT: $F \mathrm{~s}(1,7)=111.67$ and $11.05, p \mathrm{~s}<.001$ and .013 , for central and lateral targets, respectively; error rates: $F \mathrm{~s}(1,7)=7.65$ and $7.30, p \mathrm{~s}<.028$ and .031$]$ : For peripheral primes, positive compatibility effects increased in size with increasing SOA, whereas for central primes, positive compatibility effects turned into negative compatibility effects.

Follow-up analyses revealed that the main effects of compatibility at the $0-\mathrm{msec}$ SOA were significant for central targets [RT: $F(1,7)=29.83, p<.001$; error rates: $F(1,7)=12.92, p<.009]$ and approached significance for lateral targets [RT: $F(1,7)=5.28, p<.055$; error rates: $F(1,7)=5.25, p<.056]$. The prime position $\times$

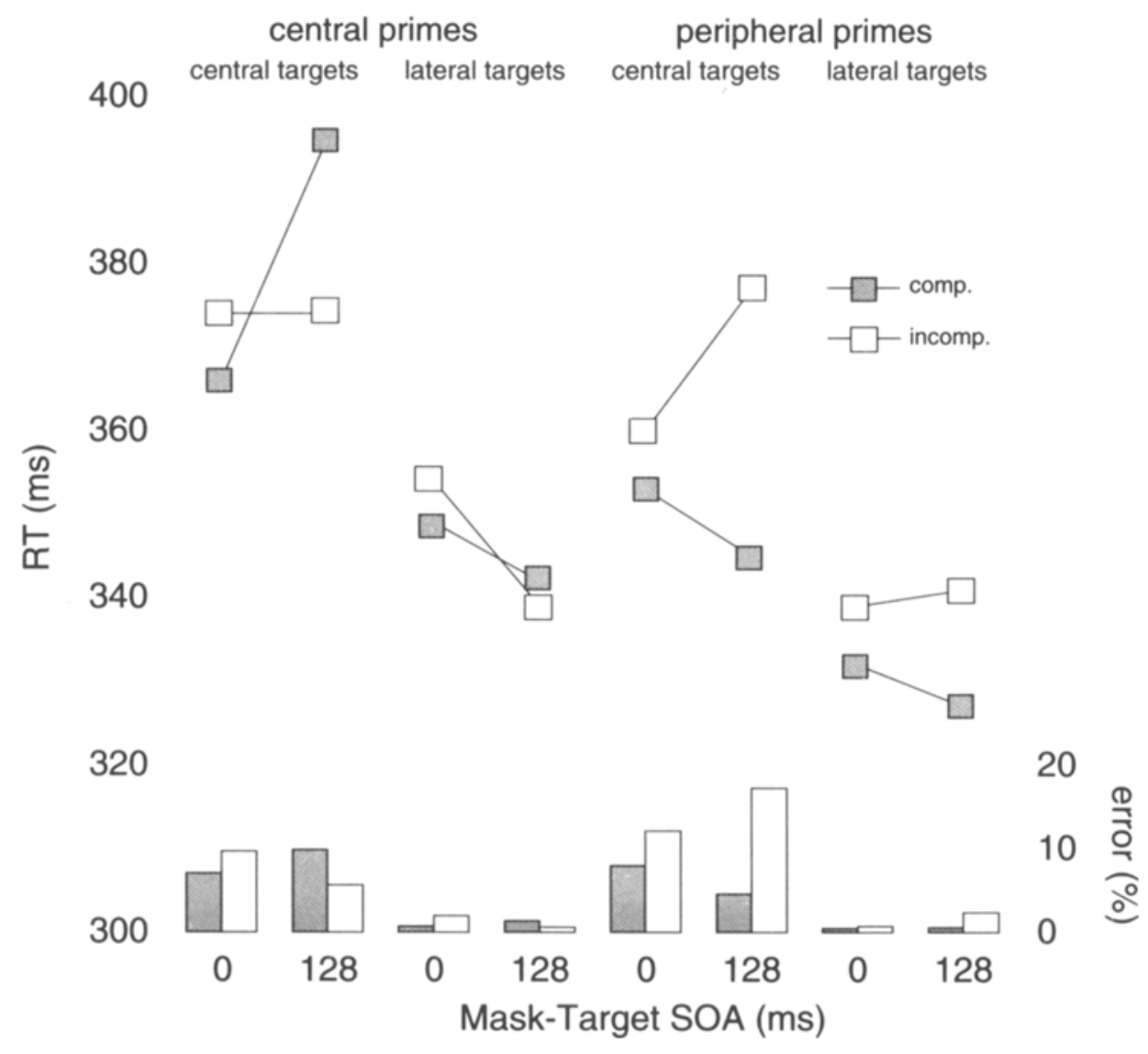

Figure 2. Experiment 2: Reaction times (RTs; line graphs) and error rates (bar graphs) in compatible trials (gray) and incompatible trials (white) in response to central and lateral targets for the 0-msec mask-target stimulus onset asynchrony (SOA) and the 128-msec mask-target SOA, displayed separately for central primes (left) and peripheral primes (right). 
compatibility interactions at the 128 -msec SOA, indicating the existence of a CPA, were significant for central as well as for lateral targets [RT: $F \mathrm{~S}(1,7)=69.22$ and $20.29, p s<.001$ and .003 , for central and lateral targets, respectively; error rates: $F_{\mathrm{s}}(1,7)=23.02$ and $7.29, p \mathrm{~s}<$ .002 and .031$]$.

\section{Discussion of Experiment 2}

Experiment 2 tested whether the CPA is caused by perceptual properties of the stimulus material (e.g., emergent visual features and/or perceptual repetition blindness effects, occurring with central primes and central targets, but not with peripheral primes and central targets), rather than reflecting systematic differences in response activation and inhibition triggered by foveal and peripheral primes. Prime-target compatibility effects elicited by central and peripheral arrow primes at short $(0-\mathrm{msec})$ and long (128-msec) mask-target SOAs on responses to central arrow targets were compared with effects on responses to lateral targets that were physically dissimilar and spatially separated from the primes. The results obtained in this experiment rule out the hypothesis that the CPA is based on the different spatial separations of physically similar primes and targets in central prime trials and peripheral prime trials: For both types of targets, positive compatibility effects were obtained with peripheral and central primes in the $0-\mathrm{msec}$ SOA condition, and a CPA was observed in the 128 -msec SOA condition.

However, some aspects of these results require further consideration. First, the size of the positive compatibility effects obtained in the $0-\mathrm{msec}$ SOA condition was considerably smaller than that in Experiment $1 \mathrm{~A}$. This may have been caused by the fact that primes appeared as targets on only $50 \%$ of all the trials. Further studies will have to investigate whether the strength of the initial response activation depends on the probability of primes being presented as targets. Second, although prime position $\times$ compatibility interactions were present for both target types in the 128-msec SOA condition, indicating the existence of a CPA for central as well as for lateral targets, inspection of Figure 2 suggests--and subsequent $t$ tests confirmed-that negative compatibility effects elicited by central primes were significant only for central targets. This could indicate that the physical similarity between primes and targets is, in fact, crucial for the presence of an inhibitory process, as is suggested by the perceptual repetition blindness account. There is, however, an alternative explanation: As can be seen from Figure 2, responses to lateral targets were considerably faster than responses to central targets. Mean RT to lateral targets was about $340 \mathrm{msec}$ - that is, responses started, on average, about $470 \mathrm{msec}$ after prime offset. This primeresponse interval corresponds almost exactly to the interval measured in the 64-msec SOA condition in Experiment $1 \mathrm{~A}$, where mean RT to central targets was about $400 \mathrm{msec}$ and no prime-target compatibility effects were obtained at all (see Figure 1, left). A facilitation-followedby-inhibition account would assume that the fastest re- sponses were selected and activated during the initial facilitatory phase and that only slower responses were subject to response inhibition.

This was tested by an analysis of RT distributions (20th, 40th, 60th, and 80th percentiles), computed for each participant individually, for the central-prime/128-msec SOA condition for lateral targets. A response latency $\times$ compatibility interaction was obtained $[F(3,21)=6.75, p<$ $.013 ; \varepsilon=.571]$, and further analyses showed significant negative compatibility effects for the 60th and 80th percentiles [both $t \mathrm{~s}(7)>2.67$, both $p \mathrm{~s}<.032$ ], whereas the trend toward a positive compatibility effect for the 20th percentile was not significant. This confirms the hypothesis that the absence of a negative compatibility effect for responses to lateral targets was due to the short latency of many responses and that this effect is present for slower responses.

Overall, the results obtained in Experiment 2 demonstrate that the presence of prime-target compatibility effects and the CPA do not critically depend on visual features of the target stimuli but, rather, reflect processes involved in response selection and activation: Whereas masked primes presented centrally trigger an activationfollowed-by-inhibition process, masked primes presented peripherally trigger an activation-only process.

\section{EXPERIMENT 3}

With an explanation in terms of emergent visual features or perceptual repetition blindness ruled out, it is still unclear why the spatial location of a masked prime should determine whether or not an inhibition process is elicited. An alternative explanation is that attentional factors are responsible for the CPA. Note that in both previous experiments, targets were presented close to fixation. Since attention is likely to be directed to the expected location of target stimuli, centrally presented primes were located within the attentional focus, whereas peripheral primes were presented at unattended positions. It is conceivable that the presence or absence of response inhibition is directly related to the position of the prime relative to the current focus of attention.

In the present experiment, the role of visual-spatial attention was studied by combining the masked prime paradigm with an exogenous spatial cuing paradigm. Spatially uninformative peripheral stimuli were presented prior to each prime-mask-target sequence. These cues were assumed to involuntarily summon visual-spatial attention to their location (Müller \& Rabbitt, 1989; Remington, Johnston, \& Yantis, 1992; Riggio \& Kirsner, 1997). Masked primes and targets were presented with equal probability at cued and uncued locations. Primes presented at cued positions should be located within the focus of attention, whereas primes presented at uncued positions should be located outside this focus. If the CPA depended on the current locus of transient visual-spatial attention, in that response inhibition was elicited for primes at attended locations but not for primes at unat- 
tended locations, a negative compatibility effect similar to the effects obtained previously with central primes should be found for trials in which peripheral masked primes appeared at cued locations, whereas a positive compatibility effect should be obtained at uncued (unattended) locations.

Note that a failure to find this pattern of results could be due to the fact that the peripheral cues were not effective in attracting visual-spatial attention. Thus, in order to have an independent estimate of the effectiveness of the exogenous attentional cuing procedure, occasional probe trials were delivered together with the usual prime trials. In probe trials, participants had to respond to leftpointing or right-pointing arrows, which appeared instead of the prime but were neither masked nor followed by an additional arrow. If the attentional cuing procedure was effective, responses to probe targets at cued locations should be faster than responses to probe targets at uncued locations. If such exogenous cuing effects were obtained on probe trials without any modulatory influence of attentional cuing on prime-target compatibility effects, this would demonstrate that visual-spatial attention is not a critical factor for the presence of the CPA.

\section{Method}

Participants. Eight paid volunteers (4 male), 23-39 years of age (mean age, 26.6 years), participated in the experiment. All the participants were right-handed and had normal or corrected-to-normal vision.

Stimuli and Apparatus. Left- and right-pointing double arrows $(<<$ and $>>)$, subtending a visual angle of approximately $1.15^{\circ} \times$ $0.4^{\circ}$, served as prime and target stimuli. The mask was constructed via superimposing these stimuli upon one another. The cue consisted of a rectangular frame of approximately $1.4^{\circ} \times 0.8^{\circ}$. In addition, a fixation cross was presented, subtending a visual angle of approximately $0.3^{\circ} \times 0.3^{\circ}$. All the stimuli were presented in black on a white background on a 17 -in. computer screen.

Procedure. The general experimental set-up was similar to Experiments 1 and 2 . To support central eye fixation, a fixation cross was continuously present at the center of the screen. The experiment started with a practice block of 160 trials. The data from this block were not stored. After the practice block, a baseline block was delivered ( 240 trials), in which no cues were presented. Finally, an attentional cuing block was delivered ( 960 trials). In each block, there was a break after every 80 trials. The participants initiated the next series of 80 trials when they felt ready to do so.

In all the blocks, $80 \%$ of all the trials were prime trials and $20 \%$ of all the trials were probe trials. Prime trials consisted of a prime arrow (16-msec duration), pointing randomly and with equal probability to the right or to the left. The prime was immediately followed by a mask (100-msec duration), which in turn was immediately followed by a target stimulus ( $100-\mathrm{msec}$ duration). Trials were termed compatible if prime and target arrows pointed in the same direction and incompatible otherwise. Both conditions were equiprobable and randomized within each block. Probe trials consisted of one double arrow only (probe arrow), which was neither masked nor followed by an additional arrow and was presented for $100 \mathrm{msec}$. The intertrial interval was $1 \mathrm{sec}$. The participants had to respond to the probe arrows in the same way that they had to respond to the prime trial targets. For any given trial, all the stimuli (prime, mask, and target in the prime trials and probe arrows in the probe trials) appeared either $3.2^{\circ}$ above or $3.2^{\circ}$ below fixation, both positions being equiprobable and randomized within each block.
In the practice block as well as in the baseline block, prime trials and probe trials were presented without a prior cuing stimulus. In the attentional cuing block, each trial started with the presentation of a peripheral cue, appearing randomly and with equal probability either $3.2^{\circ}$ above or $3.2^{\circ}$ below fixation and remaining visible for $110 \mathrm{msec}$. Either a prime trial or a probe trial (see above) was delivered $55 \mathrm{msec}$ after the offset of the cue. Masked primes and targets appeared with equal probability at cued and uncued locations.

Data analysis. For prime trials in the baseline block, two-tailed $t$ tests were computed on RTs and error rates for compatible versus incompatible trials. For prime trials in the attentional cuing block, repeated measures ANOVAs were performed on RTs and error rates for the within-subjects factors of cue validity (cued vs. uncued location), prime/target compatibility (compatible or incompatible), and response side (left or right) For probe trials in the attentional cuing block, two-tailed $t$ tests were computed on RTs and error rates for targets at cued versus uncued locations.

\section{Results}

Cue validity effects. Probe trials in the attentional cuing block showed a main effect of cue validity on RT and error rates, indicating that the cues were efficient in attracting visual-spatial attention. RTs were 441 and $471 \mathrm{msec}$ to targets at cued and uncued locations $[t(7)=$ $3.43, p<.011]$, and the respective error rates were $1.3 \%$ and $3.9 \%[t(7)=4.24, p<.004]$. Cue validity also affected RT (but not error rate) on prime trials, since responses on validly cued prime trials were about $12 \mathrm{msec}$ faster than responses on invalidly cued prime trials $[F(1,7)=6.97$, $p<.033$; see Figure 3].

Prime-target compatibility effects. In the baseline block, in which no cues were presented, performance was better on compatible trials than on incompatible trials [RT: $424 \mathrm{msec}$ vs. $445 \mathrm{msec} ; t(7)=3.91, p<.006$; error rates: $5.3 \%$ vs. $9.2 \% ; t(7)=3.15, p<.016]$. Analogous effects were found in the attentional cuing block (see Figure 3), since compatible trials produced faster RTs and fewer errors than did incompatible trials [RT: $F(1,7)=35.87, p<.001$; error rate: $F(1,7)=30.33, p<$ $.001]$. Moreover, the positive compatibility effect observed in the attentional cuing block showed a tendency to be larger on valid trials than on invalid trials, confirmed by a validity $\times$ compatibility interaction that was significant for error rate $[F(1,7)=32.42, p<.001]$ and approached significance for RT $[F(1,7)=4.63, p<.069]$.

\section{Discussion of Experiment 3}

If the response tendencies triggered by stimuli within the attentional focus are subject to inhibition, whereas no inhibition is elicited when such tendencies are caused by unattended stimuli, presenting masked primes and targets at previously cued or uncued peripheral locations should affect the direction of prime-target compatibility effects. In Experiment 3, uninformative peripheral cues were presented either above or below fixation, and all subsequent stimuli (prime, mask, and target on prime trials and probe arrows on probe trials) appeared randomly and with equal probability at the cued location or at the uncued location. If the CPA was determined by the current focus of attention, a negative compatibility effect similar 


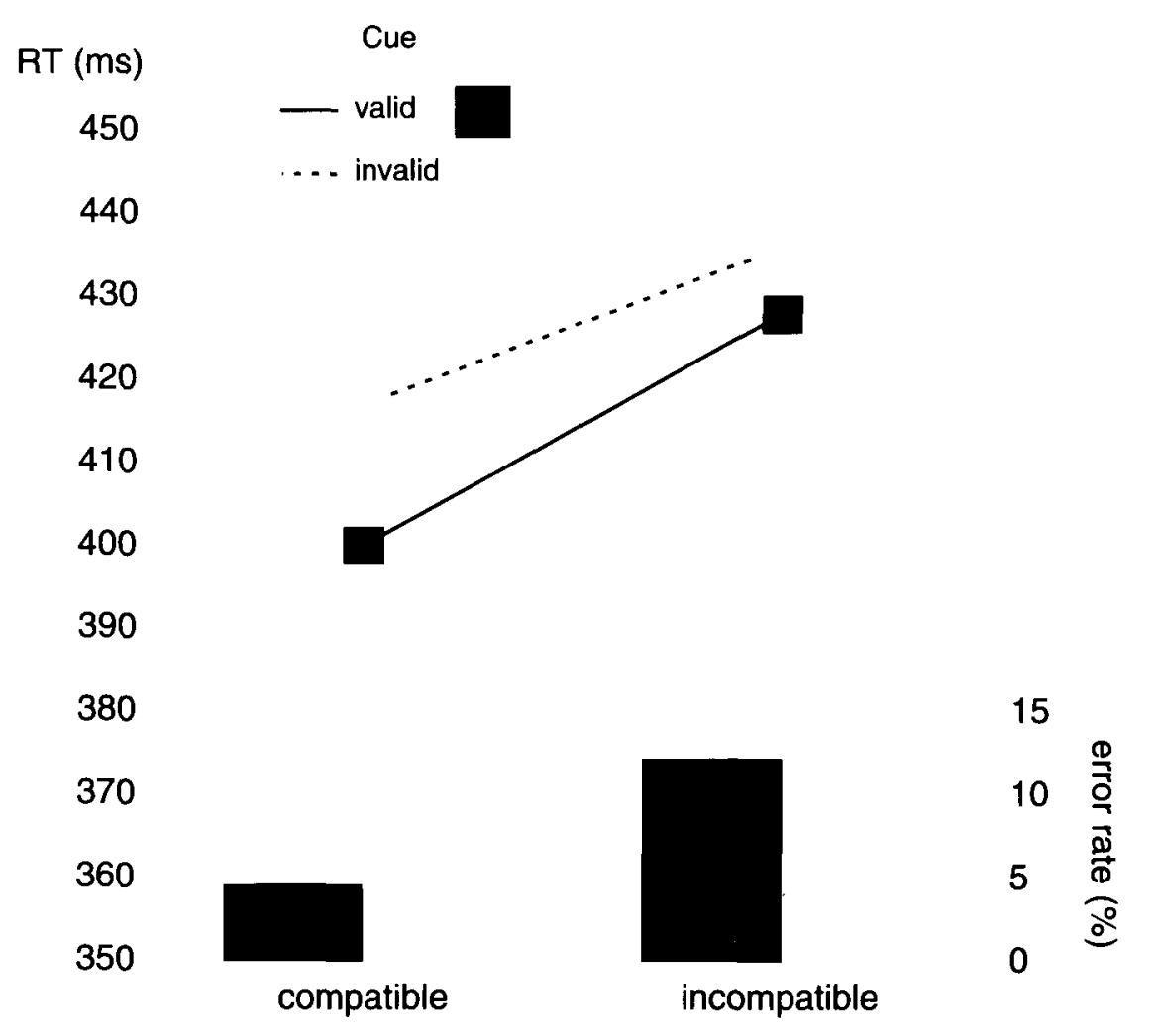

\begin{abstract}
Figure 3. Experiment 3: Performance in compatible and incompatible prime trials in the experimental block. Lines depict reaction times (RTs), bars depict error rates. Invalidly cued trials are indicated by white circles and white bars; validly cued trials are indicated by black squares and black bars.
\end{abstract}

to the effects observed with central primes should be found for masked primes and targets at cued locations, whereas a positive compatibility effect should be present at uncued locations.

The results of the present experiment do not support this assumption. Although exogenous cuing effects on RT and error rates on probe trials showed that attention was summoned to cued locations, there was no indication that a negative compatibility effect was elicited for prime trials at these locations. On the contrary, the interaction between cue validity and compatibility (only marginally significant for RT, but highly significant for error rate) reflected the opposite direction of effects: If anything, the positive compatibility effect was larger on valid trials than on invalid trials. ${ }^{6}$ Thus, one is led to assume that attentional factors play no major role for the occurrence of the CPA.

However, it has to be noted that in the present trial-bytrial cuing experiment, only the role of transient attentional shifts was investigated. In the previous experiments, targets usually appeared at fixation, thus presumably resulting in a sustained focusing of attention at the screen center. Since there is some evidence that effects of transient attention are different from effects of sustained attention (e.g., Eimer, 1996; Nakayama \& Mackeben, 1989;
Posner, Snyder, \& Davidson, 1980), the possibility remains that the location of prime stimuli, relative to a sustained focus of spatial attention, plays a role for the CPA. Alternatively, the CPA might be directly related to the retinal eccentricity of the masked primes. In Experiment 4, these issues were investigated.

\section{EXPERIMENT 4}

In this experiment, the influence of sustained visualspatial attention, as well as the relationship between the CPA and the retinal eccentricity of the masked primes, was investigated. Since the CPA was found to be unaffected by transient attentional orienting in the previous experiment, it was tested whether sustained attention has an effect on the CPA. To investigate this issue, the possibility of maintaining a narrow focus of attention in order to selectively filter out peripherally presented irrelevant information was manipulated by varying the conditions of prime presentation. In previous experiments employing peripheral primes, prime location was constant throughout an experimental block. It should be more difficult to maintain a narrow focus of sustained attention when irrelevant stimuli are presented at unpredictable locations than when their location is constant and, therefore, en- 
tirely predictable (see Allport, 1989, for the idea that the attentional filtering of irrelevant stimuli can depend on the availability and reliability of additional spatial information about these stimuli).

The participants were assigned to one of three groups. For Group A, primes were presented either centrally or unilaterally displaced from fixation, with each of 13 possible prime locations (see below) randomized within each block. This group was expected to have the greatest difficulties in maintaining a narrow focus of attention. For Group B, masked primes were presented bilaterally, thus reducing the number of possible prime locations in any given block to 7 . It was assumed that because of the greater uniformity of stimulus presentation, maintaining a narrow attentional focus would be less disrupted in this condition. For Group C, primes were presented bilaterally and prime eccentricity was held constant throughout a given block. The participants in this group were expected to have the least difficulty selectively filtering out irrelevant peripheral information.

If this between-subjects manipulation has the predicted effect on sustained attention, the participants in Group A (most diffuse spatial attention) should show the longest RTs in response to targets, whereas the participants in Group C (most focused spatial attention) should show the shortest RTs. If the CPA is sensitive to the possibility of selectively filtering out peripheral masked primes, Group A should show the smallest CPA, because with diffuse spatial attention, the difference between attended (central) and unattended (peripheral) prime locations should be relatively small. Group $\mathrm{C}$, on the other hand, should show the largest CPA, because with highly focused attention, the attentional difference between central and peripheral locations should be most pronounced. In contrast, if the CPA is not affected by sustained attention, no CPA differences should be found between groups.

Rather than being due to attentional factors, the CPA may primarily reflect physiological characteristics of the visual system. Since the perceptual sensitivity of the visual system declines with retinal eccentricity (Anstis, 1974; DeValois \& DeValois, 1988; Lie, 1980; Rijsdijk, Kroon, \& van der Wildt, 1980), a stimulus presented peripherally has a different - probably lower-representational quality than does a stimulus presented at fixation. It seems possible that the strength of perceptual representations of prime stimuli is reflected in the strength of the primed response activation and that only motor activations exceeding a certain threshold are subject to inhibition. If this were the case, it would be more likely that stimuli presented within regions of high perceptual sensitivity would eventually give rise to an inhibition process. Negative compatibility effects may thus occur with central primes because they are presented foveally, whereas positive compatibility effects may occur for peripheral primes because they are presented extrafoveally.
Following this line of argument, one may expect to find direct relationships between variations of the retinal position of the primes and the magnitude and direction of priming effects: With increasing retinal eccentricity of the prime, a decreasing negative compatibility effect should be obtained, which should turn into a positive compatibility effect beyond some critical distance from the fovea. Since the perceptual sensitivity of the retina declines faster along the vertical axis than along the horizontal axis (i.e., the area of maximal sensitivity has an elliptical shape, with the horizontal meridian determining its major axis; see, e.g., Rijsdijk et al., 1980), this critical distance should be reached at smaller eccentricities with vertically displaced primes than with horizontally displaced primes. To test this issue, masked primes either were presented at fixation or were horizontally or vertically displaced by $0.55^{\circ}, 1.1^{\circ}, 1.65^{\circ}, 2.2^{\circ}, 2.75^{\circ}$, or $3.3^{\circ}$ of visual angle.

Note that in contrast to the hypothesis that the CPA depends on physiological inhomogeneities of the retina, the idea that the CPA is due to attentional factors would not predict any difference between horizontally and vertically displaced primes in the decrease and reversal of priming effects with increasing retinal eccentricity. Although attentional selectivity generally increases with increasing retinal eccentricity of irrelevant information, this effect is generally found to be equivalent for horizontally and vertically displaced distractors, and the attentional field therefore is assumed to be circular in shape (e.g., Henderson \& MacQuistan, 1993; Hughes \& Zimba, 1987; Pan \& Eriksen, 1993).

\section{Method}

Participants. Twenty-four paid volunteers (13 male), 20-32 years of age (mean age, 23.7 years), participated in the experiment. All the participants had normal or corrected-to-normal vision, and all but one were right-handed. The participants were assigned to one of three groups (Group A, Group B, and Group C) in order of their appearance. There were 8 participants in each group.

Stimuli and Apparatus. The stimuli and apparatus were identical to those in Experiment 3, except for the absence of a fixation cross and a cue.

Procedure. The general experimental set-up was identical to those in Experiments 1 to 3, except that the target stimuli were always presented at fixation, whereas masked primes either appeared at fixation or were displaced by $0.55^{\circ}, 1.1^{\circ}, 1.65^{\circ}, 2.2^{\circ}, 2.75^{\circ}$, or $3.3^{\circ}$ of visual angle. Displacement was either horizontal or vertical, and the direction of displacement was always blocked.

For Group A, peripheral primes and masks were presented unilaterally. In separate blocks, they could appear at fixation and along the vertical meridian above or below fixation or at fixation and along the horizontal meridian to the left or right of fixation. For Groups B and C, peripheral primes and masks were presented bilaterally - that is, either above and below fixation or to the left and right of fixation, and equidistant from fixation. Moreover, for Groups $A$ and $B$, prime eccentricities $\left(0^{\circ}-3.3^{\circ}\right)$ were randomized within each block. Half of the participants in both groups received five horizontal blocks followed by five vertical blocks (each block containing 336 trials), whereas for the other half, this sequence was reversed. For Group C, prime eccentricities were blocked, and the sequence of 14 blocks (each containing 240 trials) was randomized 

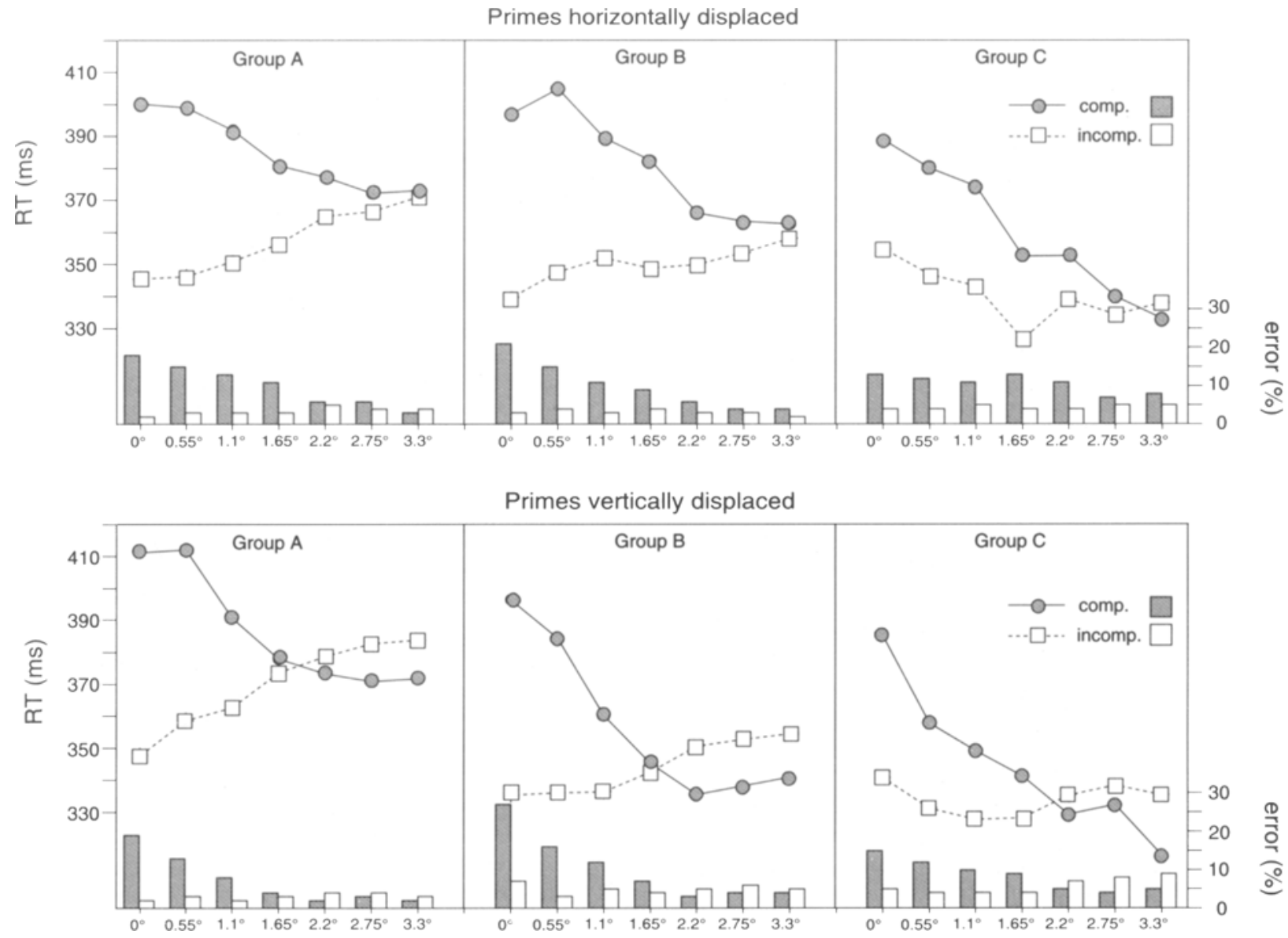

Figure 4. Experiment 4: Reaction times (RTs; lines) and error rates (bars) for compatible trials (gray) and incompatible trials (white), plotted separately for the three experimental groups. Upper panel: Blocks with horizontally displaced primes. Lower panel: Blocks with vertically displaced primes.

for each participant. There was a total of 120 trials for each of the 28 conditions (direction of displacement $\times$ eccentricity $\times$ compatibility) throughout the experiment. A rest period was included in the middle of each block, and the participants initiated the second half of the block when they felt ready to do so.

Data analysis. Repeated measures ANOVAs were performed on RTs and error rates for the between-subjects factor of group (Groups A, B, and C) and for the within-subjects factors of prime position $\left(0^{\circ}, 0.55^{\circ}, 1.1^{\circ}, 1.65^{\circ}, 2.2^{\circ}, 2.75^{\circ}\right.$, and $\left.3.3^{\circ}\right)$, direction of displacement (horizontal or vertical), and compatibility (compatible or incompatible). Where appropriate, Greenhouse-Geisser adjustments to the degrees of freedom were performed (indicated in the result section by $\mathcal{E}$ ).

\section{Results}

Prime position. Figure 4 shows RTs and error rates obtained in compatible and incompatible trials for different prime eccentricities and directions of displacement, displayed separately for the three experimental groups. RTs became generally faster and fewer errors were made with increasing eccentricity of the prime $[F(6,126)=$ $24.47, p<.001, \varepsilon=.565$, and $F(6,126)=33.83, p<.001$, $\varepsilon=.549$, respectively]. Second, RTs were faster and fewer errors were made with vertically displaced primes than with horizontally displaced primes $[F(1,21)=$ $10.35, p<.004$, and $F(1,21)=4.47, p<.047]$. These effects interacted for both RTs and error rates $[F(6,126)=$ $2.96, p<.032, \varepsilon=.571$, and $F(6,126)=5.00, p<.001$, $\varepsilon=.679$, respectively], since the performance increment with increasing eccentricities of the masked primes was more pronounced for vertically displaced primes than for horizontally displaced primes. Interactions of these effects with the factor of group are reported below.

Prime-target compatibility. Generally, RTs were faster and fewer errors were made on incompatible than on compatible trials [RT: $F(1,21)=111.95, p<.001$; error rate: $F(1,21)=61.25, p<.001$, respectively]. However, these effects became smaller with increasing retinal eccentricities of the masked primes [RT: $F(6,126)=$ $88.95, p<.001, \varepsilon=.390$; error rate: $F(6,126)=44.45$, $p<.001, \varepsilon=.383]$. Furthermore, the negative compatibility effect was generally smaller for vertically displaced primes than for horizontally displaced primes [RT: 
$F(1,21)=27.28, p<.001$; error rate: $F(1,21)=12.96$, $p<.002]$. Inspection of Figure 4 reveals that this interaction was due to the fact that with horizontally displaced primes, the negative compatibility effect decreased slowly and vanished at the largest eccentricity, whereas with vertically displaced primes, it decreased more rapidly and turned into a positive compatibility effect at $2.2^{\circ}$ [threeway compatibility $\times$ eccentricity $\times$ direction of displacement interaction; RT: $F(6,126)=10.31, p<.001, \varepsilon=$ .542 ; error rates: $F(6,126)=4.52, p<.004, \varepsilon=.570]$.

Additional $t$ tests, conducted for each prime position separately, revealed the following pattern of effects. Horizontally displaced primes caused a significant negative compatibility effect at all retinal eccentricities between $0^{\circ}$ and $2.75^{\circ}$ [all $t \mathrm{~s}(23)>2.75$, all $p \mathrm{~s}<.012$ ]. At $3.3^{\circ}$, no priming effect was present. Vertically displaced primes caused a significant negative compatibility effect at retinal eccentricities between $0^{\circ}$ and $1.1^{\circ}$ [all $t \mathrm{~s}(23)>6.01$, all $p \mathrm{~s}<.001$ ]. At $1.65^{\circ}$, only a nonsignificant negative compatibility effect was obtained $[t(23)=1.75, p<.094]$. At retinal eccentricities between $2.2^{\circ}$ and $3.3^{\circ}$, vertically displaced primes caused a significant positive compatibility effect [all $t \mathrm{~s}(23)>2.77$, all $p \mathrm{~s}<.012$ ]

Sustained attention. None of the effects that included compatibility as a factor showed any interaction with the factor of group (all $F_{\mathrm{S}}<2.2$ ). This is especially important since there was a significant main effect of group on $\mathrm{RT}[F(2,21)=4.18, p<.030]$, with Group A producing the longest RTs and Group C the shortest (mean RTs were 375,358 , and $345 \mathrm{msec}$ for Groups A, B, and C, respectively), indicating that the manipulation of prime location predictability successfully influenced attentional focusing.

Moreover, effects of eccentricity and direction of displacement interacted with group: The performance increment with increasing retinal eccentricity of the masked primes was largest for Group C, smaller for Group B, and absent for Group A [RT: $F(12,126)=5.98, p<.001$, $\varepsilon=.565$; error rates: $F(12,126)=3.89, p<.002, \varepsilon=$ $.549]$. Second, there was a group $\times$ direction of displacement interaction on RTs $[F(2,21)=12.08, p<$ $.001]$, since the RT advantage for vertically displaced primes, as compared with horizontally displaced primes, was present only for Group B and Group C, but not for Group A. Finally, there was a three-way interaction of group $\times$ eccentricity $\times$ direction of displacement interaction on RTs $[F(12,126)=2.20, p<.046, \varepsilon=.571]$.

\section{Discussion of Experiment 4}

The present results provide additional evidence that the CPA does not depend on attentional factors. The observed performance differences between the three groups showed that the manipulation of sustained attention was successful: Group A (completely randomized prime presentation) produced the longest RTs (indicating the least focused spatial attention), whereas Group C (completely blocked prime presentation) produced the shortest RTs (indicating strongly focused spatial attention). In addi- tion, Group $\mathrm{C}$ profited from increasing the distance between the target and the peripheral masked primes, whereas Group A did not, which also indicates that predictability of prime location affected the ability to selectively filter out irrelevant peripheral information. However, there was no difference between these groups with respect to the pattern of priming effects. This indicates that the CPA is not caused by differential attentional effects on the processing of central and peripheral primes under conditions in which it is possible to maintain a narrow central focus of visual attention throughout an experimental block.

The main result of the present experiment, however, is the finding of a strong dependency between the magnitude and direction of the priming effect and the retinal position of the masked primes: With central primes, the usual negative compatibility effect was observed, since RTs were faster and fewer errors were made on incompatible trials than on compatible trials. This effect was continuously reduced with increasing retinal eccentricities. Moreover, the slope of this reduction depended on the direction of prime displacement. Figure 5 shows that with horizontally displaced primes, negative compatibility effects on RTs and error rates decreased slowly and vanished only at the largest eccentricity $\left(3.3^{\circ}\right.$ of visual angle). With vertically displaced primes, the slopes of the reduction functions were steeper, reaching zero at a retinal eccentricity of approximately $2^{\circ}$ and becoming increasingly negative (i.e., the initial negative compatibility effect turned into a positive compatibility effect) with larger eccentricities.

The fact that the critical eccentricity where the negative compatibility effect turned into a positive compatibility effect was reached earlier with vertically displaced primes than with horizontally displaced primes can be taken as strong evidence for the idea that the CPA is primarily based on physiological properties of the visual system rather than on attentional factors. Although the perceptual sensitivity of the retina changes faster along the vertical axis than along the horizontal axis (Rijsdijk et al., 1980), attentional selectivity shows no such dependency (Henderson \& MacQuistan, 1993; Hughes \& Zimba, 1987; Pan \& Eriksen, 1993).

\section{GENERAL DISCUSSION}

The nature and role of inhibitory mechanisms in the adaptive control of cognitive processes and behavior have been studied intensively in a variety of paradigms, leading to the concept of inhibitory control of information processing. Usually, evidence supporting this concept has been found in studies employing suprathreshold stimuli (e.g., Kanwisher, 1987; Maylor, 1985; Raymond et al., 1992; Tipper, 1985). When stimuli are presented near detection threshold, evidence for facilitatory processes, but not for inhibitory processes, has generally been found (e.g., Allport et al., 1985; Marcel, 1980; McCormick, 1997). However, in a recent series of experiments, in 


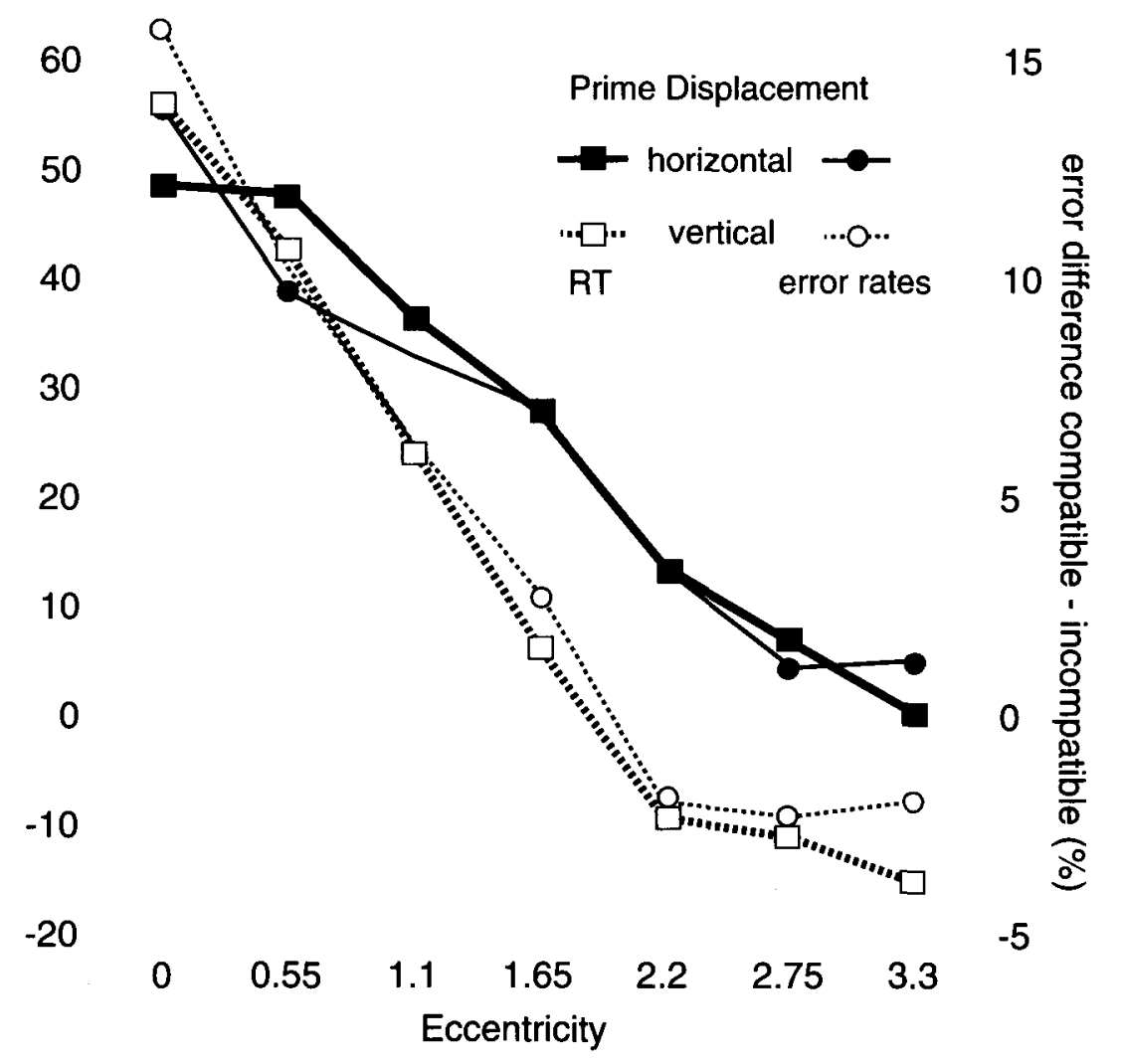

\begin{abstract}
Figure 5. Experiment 4: Mean compatible-incompatible differences in response time (RT; thick lines with square marks) and error rates (thin lines with circular marks), plotted separately for vertically displaced primes (white) and horizontally displaced primes (black). RT differences and error differences are collapsed across groups.
\end{abstract}

which target stimuli were preceded by centrally presented masked primes, evidence for the existence of inhibitory control processes under near-threshold conditions has been reported. Positive compatibility effects (better performance in compatible trials, in which primes and targets were identical, than in incompatible trials, in which they were mapped to opposite responses), occurring when the interval between prime and target presentation was short, were found to turn into negative compatibility effects (better performance in incompatible trials) when this interval was longer. It was argued that this pattern of results reflects an initial facilitation of the response assigned to the prime that is subsequently inhibited. The puzzling fact that no evidence for response inhibition was found when the masked primes were presented peripherally (CPA) suggested qualitatively different types of perceptuomotor processing for foveal and peripheral stimuli. The aim of the present series of experiments was to investigate this idea and to test alternative accounts of the CPA.

Experiment 1 tested three explanations for the presence of a CPA (delayed activation, delayed inhibition, or absent inhibition of response tendencies triggered by peripheral primes) by varying mask-target SOA between 0 and $192 \mathrm{msec}$ for central and for peripheral primes. With short prime-target SOAs ( 0 and $32 \mathrm{msec}$ ), positive compatibility effects were obtained for central and for peripheral primes. This is inconsistent with the idea that the CPA is caused by a delayed activation of the response tendency triggered by peripheral primes. With long SOAs ( $96 \mathrm{msec}$ and longer), negative compatibility effects were observed for central primes, whereas positive compatibility effects remained present for peripheral primes. This CPA remained stable within the SOA range investigated in Experiment 1 . The absence of a negative compatibility effect for peripheral primes at long SOAs contradicts the hypothesis that response inhibition is simply delayed for peripheral primes. In terms of response activation and inhibition, this pattern of results suggests that both central and peripheral primes initially trigger a partial response activation, presumably mediated by direct perceptuomotor links. With central primes, this initial activation is subsequently inhibited, and this inhibitory phase does not dissipate rapidly but remains present for at least $100 \mathrm{msec}$. No such inhibition is elicited for peripheral primes, for which the initial response tendency triggered by the prime affects performance for at least $200 \mathrm{msec}$. In other words, activation-followed-by-inhibition is restricted to centrally 
presented primes, whereas activation-only is elicited by peripheral primes.

Experiment 2 ruled out the idea that these effects are due primarily to perceptual properties of the stimuli (resulting in specific emergent features and/or repetition blindness effects), rather than reflecting response facilitation and inhibition: Positive compatibility effects for central and peripheral primes with a 0 -msec mask-target SOA, as well as a CPA with a 128 -msec SOA, were obtained not only for responses to central targets that were physically similar to the prime stimuli, but also for responses to lateral targets that were physically dissimilar from the masked arrow primes. Note that the fact that prime-target compatibility effects were elicited independent of the visual similarity between primes and targets distinguishes them from other inhibitory effects, such as repetition blindness and the attentional blink, where such similarities apparently play an important role (see Kanwisher \& Potter, 1990; Raymond, Shapiro, \& Arnell, 1995).

Together with LRP data obtained in previous studies (Eimer, 1999; Eimer \& Schlaghecken, 1998), these experiments provide converging evidence that briefly presented and subsequently masked stimuli trigger a partial response activation and that this initial response tendency is later reversed in the case of centrally presented primes. Second, and most important, the CPA cannot be explained simply by differences in the timing of effects of central and peripheral stimuli on response stages (delayed activation or delayed inhibition) but seems to reflect qualitative differences in perceptuomotor links, with inhibition restricted to response activation by foveal events.

The results from Experiments $I$ and 2 thus suggest that with respect to response activation and inhibition, foveal events are "special," but they do not provide information about what makes them special. Experiments 3 and 4 investigated the role of visual-spatial attention for the CPA: Response inhibition may have been restricted to foveal primes because attention was focused at fixation and response inhibition processes are elicited only by stimuli presented inside the focus of visual-spatial attention. We tested this by manipulating the focus of transient (Experiment 3) and sustained (Experiment 4) attention. Converging evidence was found that the CPA is not modulated by attentional factors. Neither transient shifts of focal attention triggered by exogenous trial-by-trial cuing nor differences in the focus of sustained attention related to the predictability of prime locations had an effect on the direction and size of the CPA.

Alternatively, response inhibition may be restricted to foveal primes because response inhibition processes are elicited only by stimuli presented at retinal locations of high perceptual sensitivity. Experiment 4 revealed a strong dependency between the perceptual sensitivity of the retinal region of prime presentation and the size and direction of priming effects. Generally, there was a gradual decrease in the size of negative compatibility effects with increasing retinal eccentricity of the prime, parallel to the gradual decrease of perceptual sensitivity at more peripheral retinal positions (Anstis, 1974; DeValois \& DeValois, 1988; Lie, 1980). Most important, this decrease was steeper with vertically displaced primes than with horizontally displaced primes, parallel to the faster decrease of perceptual sensitivity along the vertical meridian than along the horizontal meridian (Rijsdijk et al., 1980). This pattern of results suggests that the CPA is closely linked to variations in perceptual sensitivity: An inhibition of a response tendency triggered by a masked prime is elicited only if the prime stimulus is presented at retinal locations of high perceptual sensitivity, regardless of whether or not this location is attended.

However, these findings still leave open the question of why the retinal location of a masked prime should determine the presence or absence of response inhibition. One tentative explanation is that response inhibition processes will be initiated only when the primed response has reached an activation threshold: Given the existence of direct perceptuomotor links, strong perceptual representations may elicit stronger motor activations, which will be inhibited if they reach above-threshold values, whereas weak perceptual representations will elicit weaker motor activations, which remain below inhibition threshold. Presumably, the representational quality of sensory traces elicited by prime stimuli decreases with increasing retinal eccentricity. Consequently, motor tendencies triggered by foveal primes are more likely to be actively inhibited than motor tendencies triggered by peripheral primes. Similar ideas of a threshold determining whether gradual-accumulation-of-activation or activationfollowed-by-inhibition will take place were proposed by Hagenzieker and van der Heijden (1990; see also Hagenzieker, van der Heijden, \& Hagenaar, 1990).

Several questions are raised by this tentative idea. For example, although a strong link between CPA and physiological properties of the visual system has been demonstrated, it is unclear whether the relevant property really is perceptual sensitivity, rather than some other factor that varies with retinal eccentricity, such as, for example, temporal integration. Moreover, the threshold idea assumes that there are no qualitative differences in the processing of central and peripheral information-that is, that in either case, the processing is performed by the same visuomotor pathways. This view is not necessarily correct. For example, there is some evidence that the amount of retinal ganglion cells projecting to the superior colliculus increases with increasing retinal eccentricity (Bunt, Hendrickson, Lund, Lund, \& Fuchs, 1975; Perry $\&$ Cowey, 1984). One could therefore assume that different visual processing streams contribute differentially to the processing of central and peripheral stimuli. Whereas visuomotor systems operative in gaze shift control (e.g., the superior colliculus and related motor structures) may be more involved in processing peripheral stimuli than in processing central stimuli, even under conditions in which no overt eye movements are performed, perceptuomotor systems responsible for visuo- 
manual control may receive their input primarily from the fovea. The CPA may be related to such functional differences between visuomotor subsystems.

However, at least two predictions can be derived from the response activation threshold concept that can be tested in further studies. If the direction and time course of priming effects on performance depend on the degree to which the primed response is activated, centrally presented primes that elicit only a weak sensory trace (e.g., because of a low signal-to-noise ratio) should give rise to positive compatibility effects, reflecting the absence of response inhibition. Similarly, peripheral primes that elicit a sufficiently strong activation (e.g., owing to size scaling or longer presentation times) may trigger activation-followed-byinhibition and, thus, a negative compatibility effect.

\section{REFERENCES}

Allport, A. (1989). Visual attention. In M. I. Posner (Ed.), Foundations of cognitive science (pp. 631-682). Cambridge, MA: MIT Press.

Allport, A., Tipper, S., \& Chmiel, N. R. J. (1985). Perceptual integration and postcategorical filtering. In M. I. Posner \& O. S. M. Marin (Eds.), Attention and performance XI (pp. 107-132). Hillsdale, NJ: Erlbaum.

ANSTIS, S. M. (1974). A chart demonstrating variations in acuity with retinal position. Vision Research, 14, 589-592.

ARButhnotT, K. D. (1995). Inhibitory mechanisms in cognition: Phenomena and models. Current Psychology of Cognition, 14, 3-45.

Bunt, A. H., Hendrickson, A. E., Lund, J. S., Lund, R. D., \& Fuchs, A. F. (1975). Monkey retinal ganglion cells: Morphometric analysis and tracing of axonal projections, with a consideration of the peroxidase technique. Journal of Comparative Neurology, 164, 265-285.

COYLE, K. (1994). The relationship between time of arrival of nontargets and their spatial location: Evidence for asymmetries in visual attentional processing. Perception, 23, 849-856.

Dagenbach, D., Carr, T. H., \& Wilhelmsen, A. L. (1989). Taskinduced strategies and near-threshold priming: Conscious influences on unconscious perception. Journal of Memory \& Language, 28, 412-443.

De Jong, R., Coles, M., \& Logan, G. D. (1995). Strategies and mechanisms in nonselective and selective inhibitory motor control. Journal of Experimental Psychology: Human Perception \& Performance, 21, 498-511

DeValois, R. L., \& DeValois, K. K. (1988). Spatial vision. New York: Oxford University Press.

EIMER, M. (1996). ERP modulations indicate the selective processing of visual stimuli as a result of transient and sustained spatial attention. Psychophysiology, 33, 13-21.

EIMER, M. (1999). Facilitatory and inhibitory effects of masked prime stimuli on motor activation and behavioral performance. Acta Psychologica, 101, 293-313.

Eimer, M., \& SChlaGHecken, F. (1998). Effects of masked stimuli on motor activation: Behavioral and electrophysiological evidence. Journal of Experimental Psychology: Human Perception \& Performance, 24, 1737-1747.

ERIKSEN, B. A., \& ERIKSEN, C. W. (1974). Effects of noise letters upon the identification of a target letter in a visual search. Perception \& Psychophysics, 16, 143-149.

ERIKSEN, C. W., \& HofFmanN, J. E. (1973). The extent of processing of noise elements during selective encoding from visual displays. Perception \& Psychophysics, 14, 155-160.

ERIKSEN, C. W., PAN, K., \& Botella, J. (1993). Attentional distribution in visual space. Psychological Research, 56, 5-13.

Fox, E. (1995). Negative priming from ignored distractors in visual selection: A review. Psychonomic Bulletin \& Review, 2, 145-173.
Goolkasian, P. (1997). Size scaling and spatial factors in visual attention. American Journal of Psychology, 110, 397-415.

Gratton, G., Coles, M. G. H., Sirevaag, E. J., Eriksen, C. W., \& DonChin, E. (1988). Pre- and post-stimulus activation of response channels: A psychophysiological analysis. Journal of Experimental Psychology: Human Perception \& Performance, 14, 331-344.

Hagenzieker, M. P., \& Van der Heijden, A. H. C. (1990). Timecourses in visual-information processing: Some theoretical considerations. Psychological Research, 52, 5-12.

Hagenzieker, M. P., van der Heijden, A. H. C., \& Hagenaar, R. (1990). Time-courses in visual-information processing: Some empirical evidence for inhibition. Psychological Research, 52, 13-21.

Henderson, M. J., \& MACQuistan, A. D. (1993). The spatial distribution of attention following an exogenous cue. Perception \& Psychophysics, 53, 221-230.

Hochraus, L., \& Johnston, J. C. (1996). Perceptual repetition blindness effects. Journal of Experimental Psychology: Human Perception \& Performance, 22, 355-366.

Houghton, G., \& TipPER, S. P. (1996). Inhibitory mechanisms of neural and cognitive control: Applications to selective attention and sequential action. Brain \& Cognition, 30, 20-43.

Hughes, H. C., \& ZIMBA, L. D. (1987). Natural boundaries for the spread of directed visual attention. Neuropsychologia, 2, 5-18.

KaNWISHER, N. (1987). Repetition blindness: Type recognition without token identification. Cognition, 27, 117-143.

Kanwisher, N., \& Potter, M. C. (1990). Repetition blindness: Levels of processing. Journal of Experimental Psychology: Human Perception \& Performance, 16, 30-47.

KLotz, W., \& WoLFF, P. (1995). The effect of a masked stimulus on the response to the masking stimulus. Psychological Research, 58, 92-101.

KoPP, B., Rist, F., \& MATTLER, U. (1996). N200 in the flanker task as a neurobehavioral tool for investigating executive control. Psychophysiology, 33, 282-294.

LiE, I. (1980). Visual detection and resolution as a function of retinal locus. Vision Research, 20, 967-974.

MARCEL, T. (1980). Conscious and preconscious recognition of polysemous words: Locating the selective effects of prior verbal context. In R. S. Nickerson (Ed.), Attention and performance VIII (pp. 435-457). Hillsdale, NJ: Erlbaum.

MaY, C. P., KANE, M. J., \& HAShER, L. (1995). Determinants of negative priming. Psychological Bulletin, 118, 35-54.

MAYLOR, E. A. (1985). Facilitatory and inhibitory components of orienting in visual space. In M. I. Posner \& O. S. M. Marin (Eds.), $A t-$ tention and performance XI (pp. 189-204). Hillsdale, NJ: Erlbaum.

McCoRMICK, P. A. (1997). Orienting attention without awareness. Journal of Experimental Psychology: Human Perception \& Performance, 23, 168-180.

MILLER, J. (1991). The flanker compatibility effect as a function of visual angle, attentional focus, visual transients, and perceptual load: A search for boundary conditions. Perception \& Psychophysics, 49, 270-288.

Müller, H. J., \& RABBITT, P. M. A. (1989). Reflexive and voluntary orienting of visual attention: Time course of activation and resistance to interruption. Journal of Experimental Psychology: Human Perception \& Performance, 15, 315-330.

NakAYAMa, K., \& MaCKEBEN, M. (1989). Sustained and transient components of focal visual attention. Vision Research, 29, 1631-1647.

Neill, W. T., Valdes, L. A., \& Terry, K. M. (1995). Selective attention and the inhibitory control of cognition. In F. N. Dempster \& C. J. Brainerd (Eds.), Interference and inhibition in cognition (pp. $207-$ 261). San Diego: Academic Press.

Neumann, O., \& Klotz, W. (1994). Motor responses to nonreportable, masked stimuli: Where is the limit of direct parameter specification? In C. Umiltà \& M. Moskovitch (Eds.), Attention and performance XV: Conscious and nonconscious information processing (pp. 123-150). Cambridge, MA: MIT Press, Bradford Books.

PAN, K., \& ERIKSEN, C. W. (1993). Attentional distribution in the visual field during same-different judgments as assessed by response competition. Perception \& Psychophysics, 53, 134-144.

PerRY, V. H., \& Cowey, A. (1984). Retinal ganglion cells that project 
to the superior colliculus and pretectum in the macaque monkey. Neuroscience, 12, 1125-1137.

Posner, M. I., SNyder, C. R. R., \& Davidson, B. J. (1980). Attention and the detection of signals. Journal of Experimental Psychology: General, 109, 160-174.

Raymond, J. E., Shapiro, K. L., \& Arnell, K. M. (1992). Temporal suppression and visual processing in an RSVP task: An attentional blink? Journal of Experimental Psychology: Human Perception \& Performance, 18, 849-860.

RaYMOND, J. E., Shapiro, K. L., \& Arnell, K. M. (1995). Similarity determines the attentional blink. Journal of Experimental Psychology: Human Perception \& Performance, 21, 653-662.

Remington, R. W., Johnston, J. C., \& Yantis, S. (1992). Involuntary attentional capture by abrupt onsets. Perception \& Psychophysics, 51, 279-290.

Riggio, L., \& Kirsner, K. (1997). The relationship between central cues and peripheral cues in covert visual orientation. Perception \& Psychophysics, 59, 885-899.

RijsdiJK, J. P., Kroon, J. N., \& van DER WiLdT, G. J. (1980). Contrast sensitivity as a function of position on the retina. Vision Research, 20 , 235-241.

SCHLAGHECKEN, F., \& Eimer, M. (1997). The influence of subliminally presented primes on response preparation. Sprache und Kognition, 16, 166-175.

Smid, H. G. O. M., Mulder, G., \& Mulder, L. J. M. (1990). Selective response activation can begin before stimulus recognition is complete: A psychophysiological and error analysis of continuous flow. Acta Psychologica, 74, 169-201.

TIPPER, S. P. (1985). The negative priming effect: Inhibitory priming by ignored objects. Quarterly Journal of Experimental Psychology, 37A, 571-590.

\section{NOTES}

1. The effectiveness of the masking procedure in preventing the conscious perception of the primes was tested in numerous forced choice performance blocks in which participants had to detect the presence of a prime or identify masked primes presented either without (Schlaghecken \& Eimer, 1997) or with (Eimer \& Schlaghecken, 1998) subsequent targets. Detection and identification performances were at chance level, and participants consistently reported their inability to respond discriminatively, suggesting that the masking procedure was effective in preventing subjective awareness of the primes.
2. Experiment $1 \mathrm{~A}$ is a partial replication of Schlaghecken and Eimer (1997). In this study, positive compatibility effects obtained with peripheral primes were substantially smaller than the corresponding negative compatibility effects obtained with central primes, and 4 out of 10 participants even showed a tendency for negative compatibility effects with peripheral primes in one or the other SOA condition. Since the experiment was very demanding for the participants (consisting of 40 RT blocks and 20 different experimental conditions), we assume that this reflected less-than-perfect eye movement control (note that for primes presented at or near fixation, negative compatibility effects are to be expected). Therefore, we felt it necessary to confirm with a less demanding set-up that the CPA is a reliable phenomenon.

3. Additional forced choice detection blocks for central and peripheral primes were delivered at the end of both experiments, including masked primes $16 \mathrm{msec}$ in duration, as well as primes of longer durations, to prevent participants from switching to random guessing because of their perceived inability to perform as instructed. Detection performance for central and peripheral $16-\mathrm{msec}$ primes was at chance level, showing that the masking procedure successfully prevented conscious awareness of the primes.

4. The bilateral central targets were arranged vertically, rather than horizontally (as in Experiment 1), in order to counteract an increased tendency to move the eyes to the left or the right, revealed in a pilot study, which is most likely related to the inclusion of unilateral targets in the left or the right visual field.

5. One of the four conditions of Experiment 2 (central primes, long mask-target SOAs) is similar to a previous experiment reported by Eimer (1999, Experiment 1). A negative compatibility effect was obtained for both target types, providing initial evidence that perceptual repetition blindness is not responsible for this effect. Since no peripheral primes were used in this experiment and SOA was not varied, this experiment did not provide any direct evidence about the relationship between the physical similarity of the primes and targets and the CPA.

6. Note that this result cannot be explained as an artifact of eye movement: If participants had moved their eyes to the cued location, one should expect a negative compatibility effect for cued prime trials, because masked primes would be at or close to fixation and foveal primes are known to elicit a negative compatibility effect at the prime-target interstimulus interval employed in the present experiment (Eimer, 1999; Eimer \& Schlaghecken, 1998; Schlaghecken \& Eimer, 1997).

(Manuscript received February 9, 1999; revision accepted for publication December $15,1999$. 